\title{
Curvature in hand movements as a result of visual misjudgements of direction
}

\author{
ELI BRENNER* ${ }^{*}$ JEROEN. B. J. SMEETS \\ and H. C. M. REMIJNSE-TAMERIUS \\ Vakgroep Fysiologie, Erasmus Universiteit, Postbus 1738, 3000 DR Rotterdam, The Netherlands
}

Received 31 October 2001; revised 2 January 2002; accepted 5 April 2002

\begin{abstract}
The path that our hand takes when moving from one position to another is often slightly curved. Part of this curvature is caused by perceptual errors. We examine here whether this is so for the influence that a surface's orientation has on the approaching hand's path. When moving our hand towards a point on a surface we tend to follow a path that makes the final approach more orthogonal to the surface at that point. Doing so makes us less sensitive to imperfections in controlling our movements. Here we show that this tendency is also present when moving towards a point along an edge of a drawing of an oriented bar. The influence of the bar's orientation is no smaller when people are explicitly asked to move as straight as possible, than when they are instructed to move as fast as possible. The bar's orientation also influences perceptual judgements of a straight path, but this influence is only as large as it is on the curvature of the hand's path for judgements of the direction from the hand's initial position to the target. We conclude that the influence of the bar's orientation on the curvature of the hand's path is caused by a misperception of the initial direction in which the hand has to move to reach the target.
\end{abstract}

Keywords: Movements; curvature; path; perception and action; human.

\section{INTRODUCTION}

When moving a finger from one place to another across an uncluttered surface, the path that the finger follows is normally almost, but not quite, straight. Beside the variability one would expect from any natural movement, there are also modest systematic deviations (De Graaf et al., 1991; Wolpert et al., 1994; Miall and Haggard, 1995; Osu et al., 1997; Boessenkool et al., 1998; Van Thiel et al., 1998; Goodbody and Wolpert, 1999). It may appear intuitively obvious that paths should be curved, considering that such movements are driven by rotations around the elbow, wrist and shoulder. Boessenkool et al. (1998) showed that the curvature

\footnotetext{
*To whom correspondence should be addressed. E-mail: brenner@ fys.fgg.eur.nl
} 
when moving the left hand is a mirror image of that made when moving the right hand, suggesting that the origin of the curvature is likely to be related to the anatomy of the arms. There is reason to believe that some such curvature is planned (Osu et al., 1997), rather than being an artefact of failing to account for the dynamics of the arm, possibly representing an inclination to minimise joint rotation (Barreca and Guenther, 2001) or torque change (Nakano et al., 1999). Such curvature of our hand's paths has nothing to do with vision. However, there is also some curvature of the hand's path that does appear to originate from perceptual errors.

De Graaf et al. (1991) asked subjects to move their hand slowly in the direction of a target. The subjects' hands followed slightly curved paths. They also asked the subjects to align a rod with the target. The rod rotated around the position from which the hand had started to move. The errors in the perceptual aligning task were consistent with the direction in which the finger had started moving. They concluded that the curvature is caused by a systematic distortion of visually perceived space. In a later study, De Graaf et al. (1996) showed that the direction in which the hand initially moved was only correct if the target was on an extension of a line connecting the starting point of the hand with the subject's body midline. This was so irrespective of where the hand started. Otherwise, subjects made systematic errors in the initial direction of movement. These errors did not depend on the distance between the hand and the target, or on how far from the subject the hand started. Thus the configuration of the arm was not critical.

In accordance with the idea of a misjudgement of space that is visual in origin, Flanagan and Rao (1995) found that when visual feedback about the hand's path is deformed, subjects make curved movements to keep the visual feedback straight. Moreover, Miall and Haggard (1995) found that blind subjects make much straighter movements than blindfolded subjects with normal vision. De Graaf et al. (1994) found that congenitally blind subjects make the same errors as subjects with normal vision. A relevant difference between the studies may be that De Graaf et al. used tactile targets that subjects felt throughout the trial, providing kinaesthetic feedback about their errors, whereas Miall and Haggard used memorised targets.

We discovered another way of inciting subjects to change the curvature of their path. We found that subjects tend to approach surfaces orthogonally, even if specifically instructed to move in a straight line (Brenner and Smeets, 1995). A tendency to approach surfaces orthogonally makes sense, because it improves the tolerance to inaccuracy in executing the movement, and thereby ensures that one can really reach the desired position on the object (see Fig. 1). If one approaches a surface orthogonally, the error on the surface (the deviation of the grey finger from the black one in Fig. 1B) corresponds exactly to the deviation from the intended path at the moment that one hits the surface. In contrast, when approaching a surface at an angle, the error at contact can be considerably larger than the deviation from the intended path (proportional to $1 / \sin$ [angle of approach]). Note how the distance between the fingers when measured along the surface is twice as large in Fig. 1A than in Fig. 1B, although the distance between the paths is the same. An additional 
A

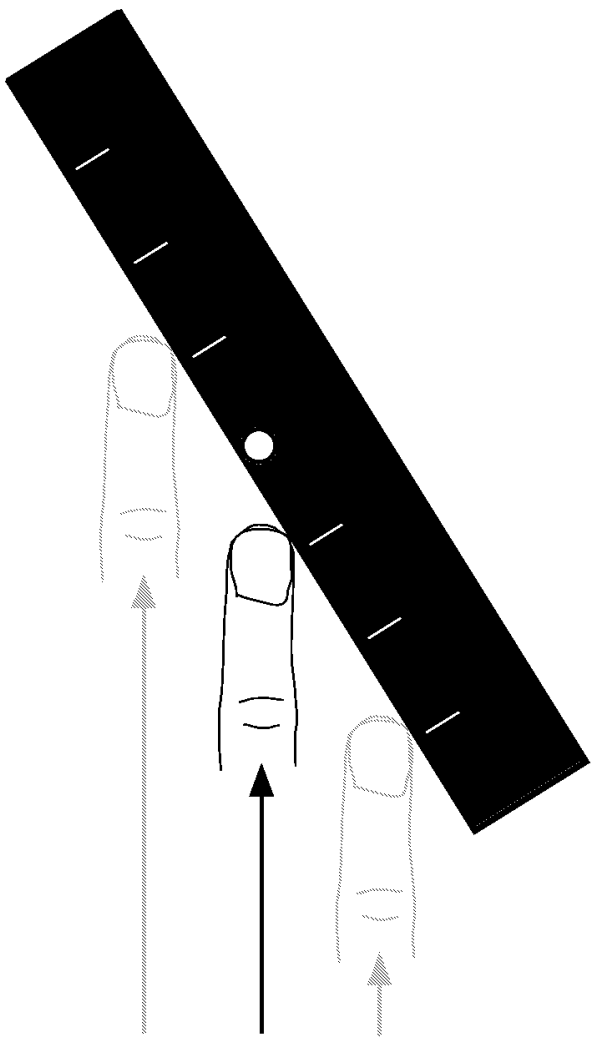

B

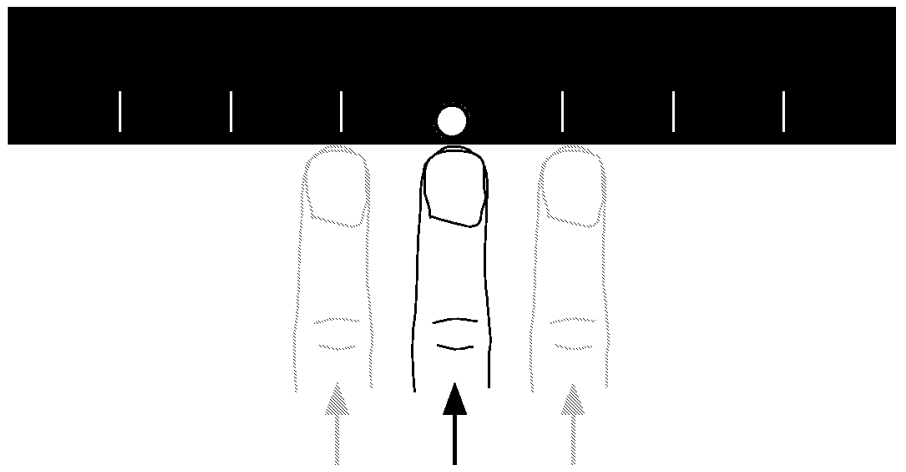

Figure 1. Illustration of why a perpendicular approach could be useful for reaching a desired position (the white dot) on an object (the black rectangle). The finger is moving straight upwards towards a slanted surface (A) or one that is perpendicular to the movement (B). Note how a lateral error in the finger's trajectory gives rise to a larger error on the object's surface if the latter is not perpendicular to the motion. (The error is inversely proportional to the sine of the angle of approach.) Moreover, a bias is introduced (in this example to the right), because the side of the finger hits the surface before the finger reaches the desired position. 
systematic error can arise because the width of the finger may make one contact the surface earlier than one had intended. An orthogonal approach also makes it less likely that the finger will slip across the surface after having made contact.

Thus, subjects may follow a slightly curved path because they seek a compromise between the instruction to move straight and the advantage that approaching perpendicularly gives with respect to reaching the target. Another possibility is that a perceptual misjudgement makes sure that subjects approach surfaces perpendicularly. The question that we set out to answer is whether subjects deviate from the path that they perceive as straight in order to ensure that they will reach the target, or whether what they perceive as straight is curved in a manner that brings about a more orthogonal approach.

\section{EXPERIMENT 1}

In the first experiment we compared the effect of target orientation on movements that were intended to be straight, with its effect on ones that were intended to be fast. When referring to target orientation we actually always mean the orientation of a black bar. The real target of the movement is a mark half way down one side of this bar, as illustrated schematically in Fig. 1 and more accurately in Fig. 2.

If subjects are inclined to make curved movements in order to arrive orthogonally, but suppress this inclination in order to adhere to the instruction to move straight, we expect that instructing subjects to move as fast as possible instead of as straight as possible will result in a stronger influence of target orientation. No longer instructing subjects to move straight means that they no longer have to suppress any inclination to move along a curved path, while moving faster provides all the more reason to approach the surface perpendicularly, because accuracy generally decreases with speed (Fitts and Peterson, 1964). In contrast, if the target's orientation influences subjects' perceptual judgements of the appropriate path, then moving faster should not make much difference. If subjects had to make a special effort to move along the perceptually misperceived path, the influence of the target's orientation might even decrease when subjects try to move as fast as possible.

Intuitively one may expect that having to move fast will cause subjects to follow more curved paths, irrespective of the target, because the dynamics of the arm becomes more important. However, previous studies have shown that the curvature hardly depends on movement speed (Nishikawa et al., 1999) but does decrease when subjects are instructed to move straight (Osu et al., 1997). We are not particularly interested in a direct comparison between movements at different speeds or after different instructions. What interests us is the difference in curvature for movements between the same points in space for different target orientations. We want to determine whether this difference is influenced by the instruction. Thus we do not compare the curvature directly, but only the differences in curvature that result from changing the target orientation. 
In our previous study (Brenner and Smeets, 1995) we used wooden blocks as targets, and subjects had to move toward the blocks with their index finger. In that case there is a real advantage in moving along a (curved) path that approaches the target's surface more orthogonally (as explained in Fig. 1). In the present experiment we got rid of the real advantage of an orthogonal approach by using drawings of rectangles as targets. Moreover, we had subjects move the tip of a pen between indicated positions, rather than their finger, so that the width of the pointer itself was no longer an issue. If the tendency to approach targets orthogonally is an adjustment to the mechanical constraints of moving a finger with non-negligible width to a real three-dimensional object, it should not be found under these conditions. If it originates from a perceptual misjudgement, it is likely to also be present when moving a pen toward drawn targets.

\section{Methods}

Subjects were requested to move a special pen (one that left no trace) between indicated positions. Their movements were measured at $204 \mathrm{~Hz}$ and a resolution of $0.02 \mathrm{~mm}$ with a digitising tablet (WACOM A2). The subjects sat comfortably (no physical restriction) straight in front of the slightly inclined (about $5^{\circ}$ ) surface of the digitising tablet. The digitising tablet was on a standard table and subjects were encouraged to adjust the height of the chair so that they could move the pen comfortably across the whole surface of the digitising tablet. They moved the special pen that is provided with the digitising tablet across its surface. The instruction was either to move as straight as possible or to move as fast as possible.

Subjects. Seven subjects took part in the experiment. Three were the authors. The other four were colleagues who were unaware of the hypotheses under study. Examination of individual data showed no conspicuous differences between the authors and the other subjects, so no distinction is made in the further analysis.

Procedure. Subjects made movements between 3 targets that were located at the corners of an imaginary equilateral triangle (see Fig. 2). The distance between any 2 targets was $30 \mathrm{~cm}$. The targets themselves were either small black dots (about $3 \mathrm{~mm}$ diameter), or white markings ( 2 by $1 \mathrm{~mm}$ ) half-way down the long edge of a 10 by $2 \mathrm{~cm}$ black bar. The dots and bars were drawn in black ink on large sheets of white paper. Each bar was orthogonal to one of the adjacent sides of the imaginary triangle. Consequently, it made an angle of $30^{\circ}$ with the other adjacent side of the imaginary triangle. Two sets of bars were used so that both bar orientations were presented at each target position.

Separate sessions were conducted for the dots (baseline) and each of the two sets of bars. For each stimulus configuration, separate sessions were conducted for two instructions: to move as fast as possible and to move as straight as possible. No instructions were given as to how accurately subjects must reach the target, 

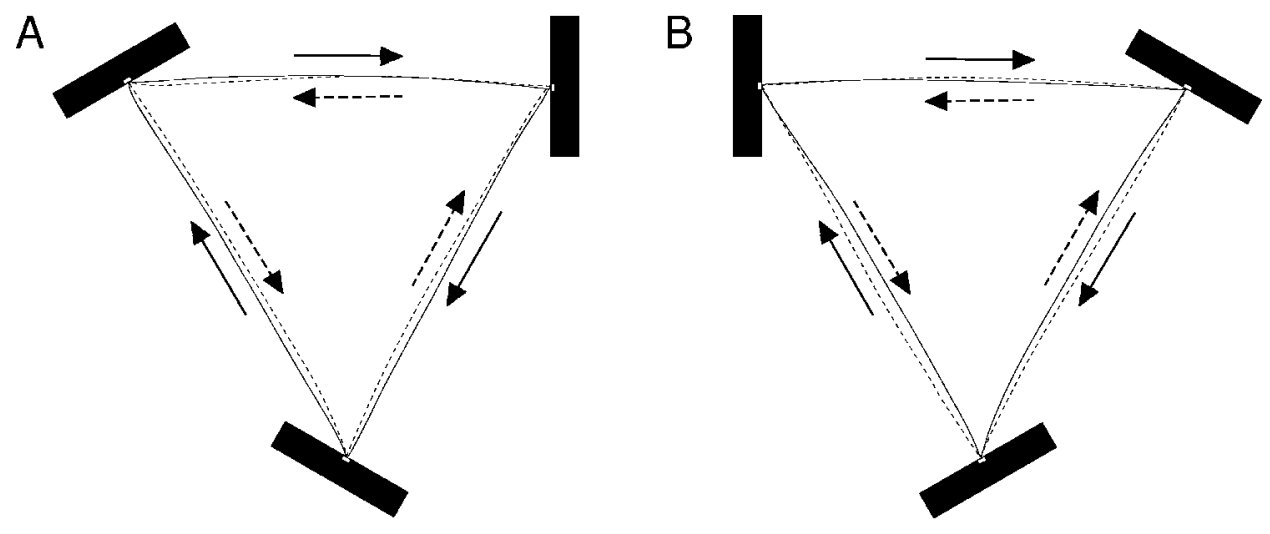

\section{Subject: DL}

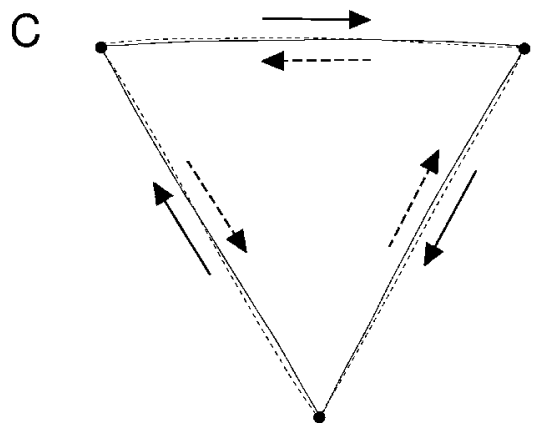

Figure 2. Example showing one subject's average trajectories for each of the stimulus configurations (A, B: bars; C: dots). The instruction was to move as straight as possible. The dashed and solid lines represent trajectories in opposite directions (indicated by the arrows). Note that the trajectories plotted as solid lines have orthogonal targets in A and slanted targets in B, whereas those plotted as dashed lines have slanted targets in A and orthogonal ones in B.

but it was explicitly mentioned that they must reach the target. The six sessions were presented in random order, with the order of the instructions counterbalanced across subjects. Each session consisted of 8 repetitions of each of the 6 possible combinations of targets, in random order. Subjects had to stop at the target, and were only told which target they should move to next after they had stopped moving.

Averaging trajectories. In order to be able to average the trajectories of movements between different combinations of targets in a meaningful manner, we defined deviations toward the centre of the imaginary triangle as positive. This ensured that whenever the bar was slanted with respect to the movement, curvature that gave rise to a more orthogonal approach was considered positive (see Fig. 2). By also using this convention for the sign of the deviation when the bar was perpendicular to the movement, and when the targets were dots, we ensured that any differences between the conditions must be due to the targets themselves.

In order to calculate average paths, each trajectory was first resampled. A straight line connecting the beginning and the end of the trajectory was divided into 100 
equal sections (101 points), and the deviation of the path from this line (the signed distance to the path in a direction orthogonal to the line) was determined at each point. Average paths were created by calculating the mean deviation for each such point, and redistributing these values along the $30 \mathrm{~cm}$ between the targets.

Statistics. The statistical analysis was based on the above-mentioned deviation of the path from a straight line connecting the beginning and the end of the movement. We rather arbitrarily chose the deviation half-way through the movement as our measure of curvature. This need not be the maximal deviation from a straight line, because the most extreme deviation is not necessarily half-way down the path. A repeated measures analysis of variance was conducted on the subjects' average half way deviation with the factors target type and instruction. This analysis was also conducted separately for each combination of targets. Our main interest was in the differences in curvature related to target type and in its interaction with the instruction given to the subject (fast or straight). A significance level of 5\% was considered throughout.

\section{Results}

When instructed to move fast, the average time it took the subjects to reach the target was $0.7 \mathrm{~s}$. When instructed to move straight it was $2.4 \mathrm{~s}$. Figure 2 shows one subject's average path for each kind of target and movement. Note the small, systematic differences (in A and B) between the two directions of motion: clockwise (solid lines) and counter-clockwise (dashed lines). Note in particular that the paths for the counter-clockwise movements deviated towards the centre in A, whereas those for the clockwise movements did so in B. These differences are consistent with a tendency to approach surfaces orthogonally. The difference cannot be related to the direction of movement, because the opposite difference between clockwise and counter-clockwise movements is found when the targets' orientations are changed (compare A and B).

Figure 3 shows average paths for each instruction, for each of the three kinds of target. Each average path includes the data of all 7 subjects and all 6 combinations of target positions. Note that the scales are not the same for the component of the movement that is in the main direction of movement, and for the component that is in the orthogonal direction. Thus, the depicted size and orientation of the slanted bar does not correspond with the scales.

The orientation of the bar clearly influenced the movement path. When the instruction was to move fast, the slanted bar gave rise to a $2.1 \mathrm{~mm}$ larger halfway deviation than the orthogonal bar. When the instruction was to move straight the half-way deviation was $3.1 \mathrm{~mm}$ larger for the slanted bar. Thus, the influence of the bar's orientation was certainly not smaller when the instruction was to move straight than when it was to move fast, although it was not reliably larger either (see next paragraph). The path toward the dots was very similar to that toward the orthogonally oriented bars. 

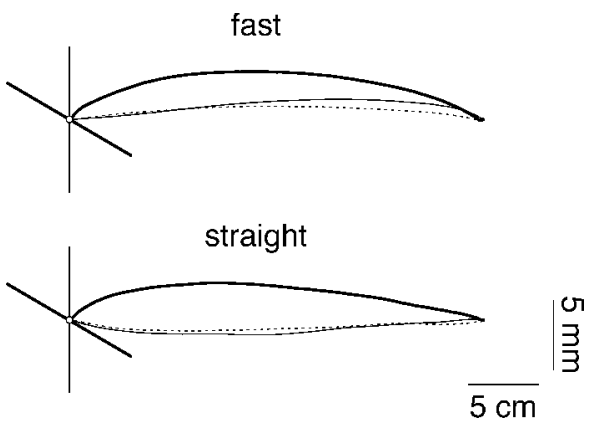

Figure 3. Overall average trajectory for each kind of target and instruction. Each trajectory is the average of 8 replications of each of the 6 trajectories for all 7 subjects (total of 336 paths). Dashed lines: movements toward dots. Thin lines: ones toward orthogonal bars. Thick lines: ones toward slanted bars. The instruction was either to move as straight or as fast as possible. For clarity, different scales are used for movements in the required direction, and ones in the orthogonal direction. Thus the depicted size and orientation of the slanted bar does not correspond with the scales.

The average standard deviation (within replications of exactly the same condition by the same subject) for the half-way deviation was $3.1 \mathrm{~mm}$ when the task was to make straight movements, and $3.7 \mathrm{~mm}$ when the task was to make fast movements. The analysis of variance showed a significant influence of target type (dot, orthogonal or slanted bar; $p<0.0001$ ) on our measure of curvature. The instruction (fast or straight) also had a significant main effect $(p=0.002)$ : the halfway deviation was on average $1.8 \mathrm{~mm}$ larger when the instruction was to move fast. This means that subjects tended to curve toward the centre of the imaginary triangle when moving fast. Why this should be so is not clear, but it demonstrates that the paths themselves were influenced by the instruction. The interaction between target type and instruction was not significant, indicating that the instruction did not change the influence of the bar's orientation.

The influence of target type was also significant for each movement path on its own, with the exception of movements from the far left to the far right stimulus (for which $p=0.052$ ). During the latter movements the subjects' arms concealed a large part of the path, and probably sometimes even of the target. This is also the only condition in which there was a significant interaction between target type and instruction $(p=0.049)$. There was a significant influence of instruction for movements from the far right to the near target, and from the near target to the far right one $(p<0.001)$, but not for any of the other movements.

\section{Discussion}

The most important findings are that the tendency to approach surfaces orthogonally is present when the targets are drawings (which cannot physically constrain the path), and that the tendency is no larger when instructed to move fast than when instructed to move straight. We conclude from this, in accordance with the reasoning in the introduction, that the influence of target orientation on the hand's 
path is mediated by a perceptual misjudgement, and is not the effect of a motor strategy.

Being mediated by a perceptual misjudgement does not automatically preclude a role in improving the tolerance to inaccuracy when executing movements. A misperception of spatial relationships could guide our finger along a curved path toward the selected position on a surface, thereby ensuring that the finger arrives orthogonally, and therefore with higher precision. However, if the movements that we found are a true representation of what we perceive to be the correct path, then the postulated misperception is unlikely to make a difference to the accuracy with which we reach real objects, because the curvature is too small. Thus the conclusion that the orthogonal approach has a perceptual origin appears to imply that it has nothing to do with accuracy. The obvious alternative, that the influence of target orientation was exceptionally small for our targets because they were only drawings, is unlikely because the curvature that we previously found with real objects was not conspicuously larger (Brenner and Smeets, 1995). Considering the potential benefits of arriving orthogonally (see Fig. 1), we do not want to exclude the possibility that trajectories toward real surfaces would curve more if the instruction were to move fast rather than straight, or if subjects had to do something at the target position (e.g. to press a button). However, contrary to the curvature in the current study, such curvature could not be attributed to a perceptual misjudgement.

If the orientation of the bar influences what subjects consider to be a straight path, we would expect to find similar misjudgements in other tasks. However, this is only so if the same notion of 'straightness' applies. There is considerable evidence against the existence of a single perceived space. Various attributes are judged independently, and the judgements are not necessarily consistent. Such inconsistencies have been found for position and extent (Gillam and Chambers, 1985; Mack et al., 1985), for displacement and change in position (Abrams and Landgraf, 1990), for motion and change in position (Brenner et al., 1996), for egocentric and relative positions (Brenner and Cornelissen, 2000), and so on. Thus, the misjudgement need not be present in all attributes. Does this mean that it is pointless to try to find the same effect in another task? We believe not, because by comparing the misperception across tasks we can hope to find the specific attribute that is affected by the target's orientation. This will tell us what kind of information is used to guide the movement of the hand. In the hope of finding this out, we conducted a second series of experiments $(2 \mathrm{~A}-2 \mathrm{E})$.

\section{EXPERIMENT 2A}

In order to compare different tasks it is useful to standardise various aspects that could influence performance. An aspect of the task that we had not yet considered, but which could have influenced the spatial misjudgements, is where subjects directed their gaze (Mohrmann-Lendla and Fleischer, 1991). Informal questioning of the subjects revealed that some subjects believed they looked at the target 
throughout the movement, whereas others claimed to have looked back and forth between the target and the pen. For a comparison between tasks it would obviously be beneficial to standardise this to some extent. For similar reasons it would be beneficial to standardise the movement time.

In our next experiment we therefore had subjects perform a similar task as in Experiment 1 with two different instructions about where to direct their gaze. In both cases we asked them to move straight to the target in a single movement that took about 1 second. In order to limit the number of trials all movements were in the same direction and we no longer included dots as targets. Experiment 1 had shown that paths towards isolated dots were similar to ones toward 'orthogonal' targets. In order to increase the differences between the paths toward different targets we therefore replaced the dots by bars that were slanted in the opposite direction. Experiment 1 had also shown that the influence of target orientation was not restricted to certain directions. We therefore chose a direction of motion that ensured that the hand would never occlude the target (from right to left for righthanded subjects).

\section{Methods}

The task was to make movements in a single direction between two dots (see Fig. 4). Subjects were always instructed to move as straight as possible, but were told that they had to reach the target. The amplitude of the movement was reduced to $20 \mathrm{~cm}$ because of the limited size of the computer screen in the perceptual tasks that were to follow. The starting position was a black dot $(2.5 \mathrm{~mm}$ diameter). The target was

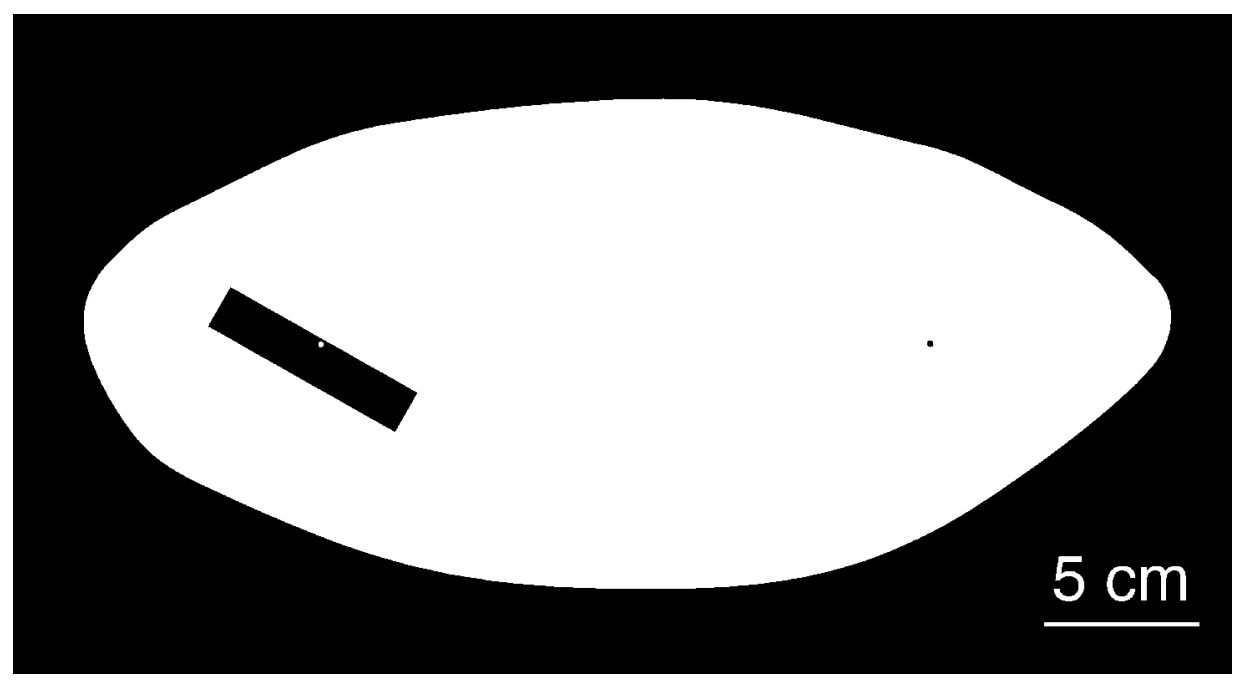

Figure 4. Example of a stimulus used in Experiment 2A (slanted bar). The subject moved the pen from the black dot to the white one. In Experiments $2 \mathrm{~B}$ to $2 \mathrm{E}$, the subject either set the path of an additional dot that moved from the black to the white dot, or set the curvature of a line connecting the two, to appear straight. 
a white dot half way down the long edge of a 70 by $14 \mathrm{~mm}$ black bar. An irregular black mask surrounded the targets and the space between them. The bar could be oriented $30^{\circ}$ upwards relative to the movement path (as in Fig. 4), 30 downward relative to the movement path, or orthogonal to the movement path (see symbols at the bottom of Fig. 5). The black mask was the same on all trials, and its position was fixed. The starting position and the position of the target were varied slightly between trials by not always placing the sheet of paper on which they were drawn at exactly the same position, or with precisely the same orientation, on the digitising tablet.

As in Experiment 1, subjects sat comfortably in front of the digitising tablet. They were asked to take about one second for each movement. They received verbal feedback about their movement time. The different orientations of the bar were presented in random order. Each subject first made 30 movements (10 for each orientation of the bar) while trying to keep looking at the pen during the movement (they were encouraged to look at the target before starting to move), and then another 30 while fixating the target.

Ten subjects, which included two of the authors, took part in the experiment. For most subjects the movements were from right to left across the body midline. One subject was left-handed. He was instructed to move in the opposite direction to the others, across a mirror-imaged scene, and the data were mirrored for analysis. Examination of individual data showed no conspicuous differences between the authors and the other subjects, or between the left- and right-handed subjects, so no distinction is made in the further analysis.

\section{Results}

The average (resampled) paths are shown in Fig. 5A. Subjects tended to approach the bar orthogonally irrespective of the viewing instructions. A repeated measures analysis of variance with viewing instruction and bar orientation (all 3 possibilities) as factors revealed a significant influence of bar orientation $(p=0.01)$, but not of viewing instruction $(p=0.27)$, and no significant interaction between the two $(p=0.70)$. The overall average influence of target orientation on the deviation half-way through the movement was $1.072 \mathrm{~mm}$ (difference between the two kinds of slanted targets).

\section{Discussion}

Where subjects were looking during the movement did not make much difference. The influence of the bar's orientation appears to be smaller than in Experiment 1. In this experiment we compare two oppositely slanted bar orientations (Fig. 5A), whereas the comparison in Experiment 1 (Fig. 3) was between a slanted bar and one that was orthogonal to the movement. We would therefore expect to have a larger effect here, which we do not. This is probably largely due to the reduced amplitude of the movement. Other factors that could contribute to the difference include the 
A

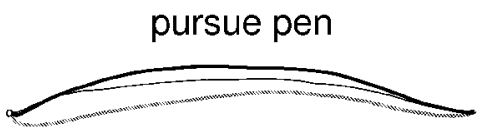

B set line / free viewing / cc

set dot path / pursue dot / cc fixate target

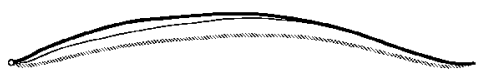

set line / fixate target / cc

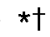

set dot path / fixate target / cc

set line / fixate target / cc $\dagger$

set dot path / fixate target / cc

set dot path / pursue dot / Cc

set line / fixate target

D set line / free viewing

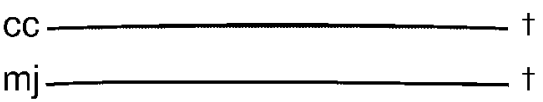

set dot path / pursue dot

set dot path / fixate target
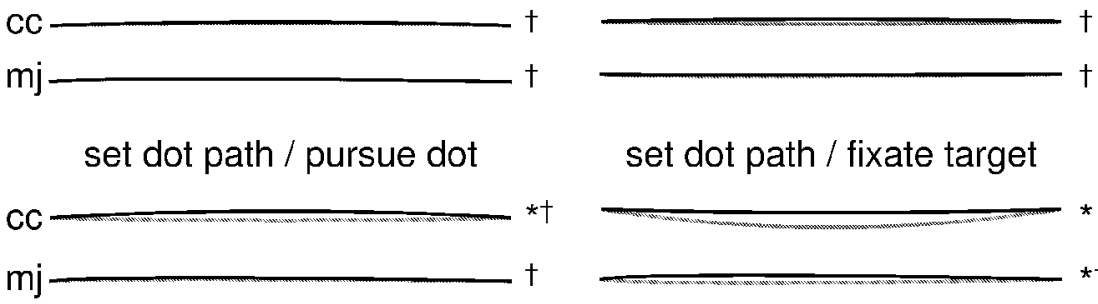

*十

\section{E $2 \mathrm{~cm}$ line / free viewing / $\mathrm{mj} \quad 2 \mathrm{~cm}$ dot path / free viewing / $\mathrm{mj}$}

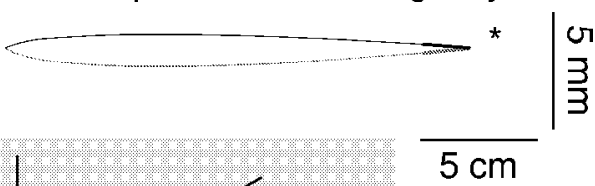

* significant influence of target orientation

$\uparrow$ significantly different from average influence in $A$

Figure 5. Results of Experiments $2 \mathrm{~A}$ to $2 \mathrm{E}$ : averages of 10 subjects. The coding of the paths for the three different orientations of the target is shown at the bottom of the figure. The symbols indicate whether the difference between the curvature for the two slanted target orientations was significant (*) and whether it deviated significantly from the $1.072 \mathrm{~mm}$ found in Experiment $2 \mathrm{~A}(\dagger)$. In $2 \mathrm{E}$, only the thick parts of the trajectories were visible. cc: constant curvature trajectory. mj: minimal jerk trajectory. 
fact that subjects always moved in the same direction, the direction itself, the bars being slightly smaller, and the restriction on movement time.

\section{EXPERIMENT 2B}

An obvious next step was to try to determine whether what subjects perceive as a straight path is influenced in the same manner. The most straightforward way to do so would have been to show subjects their hands' paths and ask them to judge whether they were straight. However, we do not expect all the curvature in the paths to be related to the proposed misperception of space (Osu et al., 1997; Boessenkool et al., 1998). Moreover, the outcome of such an approach would be difficult to interpret, because it is not clear how straight the path should appear to be. All the movements were approximately straight.

We therefore decided to ask subjects to set a curve on a computer screen so that it appeared to be perfectly straight (while we varied the orientation of a bar at one end). We also asked them to set the path of a dot that moved from the hand's starting position to the target so that it appeared to be straight. The latter task is slightly more difficult for the subjects, but the path of a moving dot may be more appropriate for comparison with a movement of the hand (Wolpert et al., 1994). Comparing the effect of bar orientation on the two kinds of settings may help specify the attribute that is affected by the bar's orientation, as may an evaluation of the influence of instructing subjects on where to look.

\section{Methods}

Stimuli were presented on a white computer screen (Trinitron; $120 \mathrm{~Hz} ; 39.2 \times$ $29.3 \mathrm{~cm}$; spatial resolution of $815 \times 611$ pixels, refined with anti-aliasing techniques). The two dots and the bar were identical to those of the paper stimuli used in Experiment 2A (and shown in Fig. 4). They were shifted and rotated together between trials, simulating the variability in the positioning of the paper in Experiment 2A. This prevented subjects from using local slopes or imperfections of the monitor to perform the task. The same black mask that was used in Experiment 2A was placed in front of the computer screen.

Apart from the structures shown in Fig. 4, the stimulus also contained either a black curved line that connected the two dots, or a black dot that moved between them. The curvature of the line or of the dot's path could be manipulated by moving the computer mouse. The curved line and the dot's path had a constant radius of curvature (i.e. they were segments of circles with variable diameters). Moving the computer mouse changed the radius of curvature in such a way that it resulted in a linear change in the amplitude of the deviation from a straight line (between -2.5 and $2.5 \mathrm{~cm}$ ).

When the stimulus was a (curved) line, it remained visible until the subject indicated that it was straight by pressing a button. When it was a moving dot, it 
took the dot 1 second to move (at a constant speed) from its starting position to the target. It did so repeatedly (with a $0.5 \mathrm{~s}$ interval between movements) until the subject indicated that its path was straight by pressing the button. Subjects sat facing the monitor at a distance of about $50 \mathrm{~cm}$. Again, they were not restricted physically, but they were not allowed to move appreciably nearer to or further from the screen.

The same 10 subjects as in Experiment $2 \mathrm{~A}$ each performed the two abovementioned tasks (line and dot) under two viewing instructions. The three different orientations of the bar were presented in random order. The different conditions were presented in a fixed order: adjusting a line until it was straight with no restrictions on gaze; adjusting the line until it was straight while fixating the end with the bar; adjusting the path along which a dot was moving while pursuing the dot with one's eyes; adjusting the dot's path while fixating the target. Each orientation was presented 10 times in each condition.

\section{Results}

The average settings that were considered to be straight are shown in Fig. 5B. It is evident that the curvature is different from that in the subjects' movements (Fig. 5A). At least part of the difference is not related to the bar's orientation. However, our statistical evaluation is restricted to the difference between the paths for the two slanted bar orientations. Two separate sets of $t$-tests were conducted. The input was the difference between the average half-way (i.e. maximal) deviation from a straight line for the two orientations. This was determined separately for each subject. We first tested whether these differences were significantly different from zero (i.e. whether the target's orientation influenced the settings). This was so for all four conditions, despite the modest values. The differences between the half-way deviations were $0.17,0.30,0.37$ and $0.83 \mathrm{~mm}$ for the four conditions (in order of presentation). Next, we tested whether these differences were significantly different from $1.072 \mathrm{~mm}$ (i.e. whether the target's orientation influenced the settings differently than in Experiment 2A). This was so for three of the four conditions. It was only not so when setting the dot's path while fixating the target. Finally, we determined the standard deviation in the settings for each subject, task and target orientation. The average standard deviation was $1.6 \mathrm{~mm}$ when fixating the target while setting the dot's path, $0.8 \mathrm{~mm}$ when pursuing the dot, and 0.5 and $0.3 \mathrm{~mm}$ when setting the line while fixating the target or looking where one pleases, respectively.

\section{Discussion}

The fact that we could find an orthogonal bias in purely visual tasks supports the idea that the orthogonal bias in the movements is caused by a perceptual misjudgement. However, the effect was considerably smaller in these tasks than when trying to move the pen in a straight line. The only condition for which the effect of target orientation was not significantly different from that in Experiment $2 \mathrm{~A}$ was when 
setting the dot's path while fixating the target. The influence of the target was largest in this condition, but it was still only $77 \%$ of that in Experiment 2A. Subjects' settings were also most variable in this condition. Moreover, the influence of the target's orientation was much smaller when pursuing the dot with their eyes, whereas where subjects were looking made little difference in Experiment 2A. We therefore decided to examine why the perceptual effect was smaller in some conditions.

\section{EXPERIMENT 2C}

One difference between the two preceding experiments was that the stimulus for the perceptual judgements (in Experiment 2B) was in a frontal vertical plane, whereas the movements (in Experiment 2A) were made on an almost horizontal plane. In order to examine whether this was critical, we repeated Experiment $2 \mathrm{~B}$ with the monitor surface oriented horizontally at approximately the same position relative to the subject as the tablet in Experiment 2A.

\section{Methods}

Everything was the same as in Experiment 2B except that the monitor was oriented horizontally. The monitor's screen is cylindrical. It was therefore possible to orient the screen in such a manner that the path was hardly affected by the curvature of the screen. This was important because subjects looked at the screen from an angle. It was achieved by placing the path more or less along the axis of the cylinder. The random rotations that we introduced to prevent subjects from simply reproducing the same settings were too small $(0.5 \mathrm{deg}$ at most $)$ to give rise to appreciable curvature, and did not differ systematically between the conditions.

\section{Results}

The results (mean of 10 subjects) are shown in Fig. 5C. The orientation of the screen surface may have made a difference, in particular when setting the dot's path while pursuing the dot. On the horizontal screen the set path curves away in a similar manner to the hand's movements in Experiment 2A (Fig. 5A). More importantly though, the influence of the target's orientation was not very different. The difference between the half-way deviations for the two slanted bar orientations was $0.31 \mathrm{~mm}$ when adjusting the line with no restrictions on gaze, and $0.26 \mathrm{~mm}$ when doing so while fixating the end at the bar. It was $0.66 \mathrm{~mm}$ when adjusting the dot's path while pursuing it with one's eyes, and $1.02 \mathrm{~mm}$ when doing so while fixating the target. The difference between the line and dot settings was even clearer than in Experiment 2B (Fig. 5B). Target orientation did not influence the line settings significantly when fixating the target, while the influence of target orientation was not significantly different from $1.072 \mathrm{~mm}$ (the mean value in Experiment 2A) 
for either of the dot settings. A direct comparison (additional $t$-tests) between the influences of target orientation for the two screen orientations (comparing Experiments 2B and 2C) was not significant for any of the four conditions.

\section{Discussion}

Although the bar's orientation did appear to have a slightly stronger influence at this screen orientation, this could not be confirmed statistically. Even if the orientation of the display does affect the results, it does not appear to be the main cause of the difference between the influence of target orientation on the movements (Experiment 2A) and on the perceptual judgements (Experiment 2B). The origin of the difference therefore has to be sought elsewhere.

\section{EXPERIMENT 2D}

In the preceding experiments we ignored some differences between the perceptual judgements and the movement tasks. Most conspicuously, we ignored the fact that the shape of the paths was different. From the paths of the pen in Figs 3 and 5A it is evident that the maximal deviation is closer to the target than it would be for a constant curvature. This could mean that the misperception of space is largest near to the target, which would not be too surprising since it is caused by the target. The dot also moved at a constant speed, which is certainly not representative of normal hand movements. In order to examine whether these issues are critical we repeated Experiment 2B, both with the same trajectories as we used originally (constant curvature; constant velocity) and with more natural paths and a more natural velocity profile for the moving dot. To limit the number of trials we included only the two slanted bar orientations.

\section{Methods}

For each task, each target orientation was now presented 20 rather than 10 times. Of these 20 presentations, 10 were identical to those in Experiment 2B (CC: constant curvature; constant speed), whereas the paths and velocity profiles of the other 10 were defined by a minimal jerk trajectory (MJ; Flash and Hogan, 1985) with a final acceleration orthogonal to the orientation of the bar (Smeets and Brenner, 1999). The value of the final acceleration was varied to obtain the desired curvature. This results in trajectories with a maximal deviation nearer to the target (see thin curves in Fig. 5E), resembling those of the arm movements (Figs 3 and 5A). For both kinds of trajectories the maximal deviation from a straight line was related linearly to the position of the computer mouse. Moreover, in both cases the moving dot took 1 second to reach the target. The different orientations of the bar and the different kinds of trajectory were presented in random order. The different conditions were presented in the same fixed order as in the preceding experiments. The screen was vertical, as in Experiment 2B. 


\section{Results}

The results (mean of 10 subjects) are summarised in Fig. 5D. For the original condition (CC) the results were very similar to those found in the previous experiments. The only conspicuous difference in comparison with the results of Experiment 2B (which included the very same conditions) was that the influence of target orientation was not significant for the line settings. The difference between the half-way deviations for the two slanted bar orientations was $0.08 \mathrm{~mm}$ when adjusting the line with no restrictions on gaze, and $0.30 \mathrm{~mm}$ when doing so while fixating the end at the bar. It was $0.54 \mathrm{~mm}$ when adjusting the dot's path while pursuing it with one's eyes, and $0.76 \mathrm{~mm}$ when doing so while fixating the target. For the new, more natural condition (MJ) the influence of target orientation was certainly not larger. In this condition, the target's orientation only had a significant influence when subjects set the dot's path while fixating the target. The average half-way deviations were $0.00,0.09,0.11$ and $0.46 \mathrm{~mm}$ for adjusting the line with no restrictions on gaze, doing so while fixating the end at the bar, adjusting the dot's path while pursuing it with one's eyes, and doing so while fixating the target, respectively.

\section{Discussion}

Making the path more similar to the movements of the pen decreased the influence of target orientation. This suggests a possible reason why we had been getting smaller responses in the perceptual tasks. We had assumed that subjects would perform the perceptual tasks on the basis of the global shape of the entire path. That is why we took the half-way deviation from a straight line, rather than for instance the maximal local curvature, as our measure of the magnitude of the effect of target orientation. For the same half-way deviation, a more natural trajectory will have a larger maximal local curvature, because the curvature is not spread evenly across the path. Since the influence of the target's orientation was smaller when the trajectory was more natural we suggest that detecting local curvature limits the influence of target orientation. This is consistent with the influence of target orientation being smaller for the line than for the moving dot, because local curvature is presumably easier to judge for the line. The curvature of the line can be judged from instantaneous retinal information, while the curvature of the dot's path must be judged from the changes in the orientation of the eyes during pursuit or from changes in the direction of retinal motion. An implication of this interpretation is that the misjudgement cannot be of space in general (in which case curvature should also be misjudged), but must be of a specific attribute that is essential to the movement task. We propose that it is specifically the direction to the target that is misjudged. 


\section{EXPERIMENT 2E}

In order to move in a straight line toward the target one must constantly move in the direction of the target. However, factors other than the direction to the target may dominate some perceptual tasks. For instance, subjects could set the line by looking for curved sections along the path, and making adjustments to eliminate such local curvature. In the movement task such a strategy would not work, because the largest local curvature in the hand's path is typically found when the hand has already passed its maximal deviation. Thus, even if the error is noticed, the hand has to adjust its path in order to reach the target. An equivalent perceptual task would therefore be one in which only the initial part of the path is involved (as in De Graaf et al., 1991).

\section{Methods}

To examine whether it is likely that it is the direction toward the target that is misjudged we conducted a final experiment in which subjects saw only the first part of the trajectory. The conditions were very much like those in the previous experiments, but only the first $2 \mathrm{~cm}$ of the line or of the dot's path were visible. It was not practical to have subjects try to perform this task while fixating the target, so they were always free to look where they pleased. There were therefore only two tasks, each with two target orientations. Each was repeated 20 times. The paths and the dot's velocity were defined by a minimal jerk trajectory (as in D). The halfway deviation from a straight line was still used to quantify the influence of target orientation, although this portion of the trajectory was never visible.

\section{Results}

The results (mean of 10 subjects) are summarised in Fig. 5E. The influence of the target's orientation on the half way deviation from a straight line was $0.85 \mathrm{~mm}$ for the line and $1.33 \mathrm{~mm}$ for the moving dot (average $1.09 \mathrm{~mm}$ ). In both cases the influence of the target's orientation was significant, and not significantly different from $1.072 \mathrm{~mm}$ (the average influence in Experiment 2A). Moreover, the difference between the settings for the line and for the moving dot was much smaller than in the previous perceptual experiments (Experiments 2B-2D). This is what we would predict if the influence of target orientation is mediated by a misjudgement of direction, and was limited by detectable local curvature in the previous experiments.

\section{Discussion}

We conclude that the tendency to approach surfaces orthogonally originates in a misperception of the direction in which the hand should move. Experiment $2 \mathrm{E}$ is reminiscent of the simplest variations of the Poggendorff illusion, consisting of only two lines (Fig. 1D in MacKay and Newbigging, 1977). In that case subjects had to judge where an extension of one of the lines would intersect the other. The errors 
that subjects made are consistent with judging the orientation of the line that was to be extended as being more orthogonal to the line that was to be intersected than it really was. Our study shows that the angle at which the extension of one line would intersect another can influence the judged orientation of the line that is to be extended even if they are $20 \mathrm{~cm}$ apart.

The equivalence between the perceptual effect in the present direction judgement task and the effect in the movement task (Experiment 2A) is consistent with the findings of De Graaf et al. (1991). Their study examined systematic biases that depend on the target's position in space. They too found equivalent errors for perceptual judgements of the direction to a target (a pointer setting task) and for the initial movements of the hand toward that target.

\section{GENERAL DISCUSSION}

Although we plot average paths in Figs 3 and 5, our statistical analysis is always based on the difference between individual subjects' half-way deviations for targets on differently oriented bars. The influence of the bar's orientation on the hand's path is quite modest. For $20 \mathrm{~cm}$ movements we only found differences in the halfway deviation of about $1 \mathrm{~mm}$. Nevertheless these differences were very consistent across subjects, so they cannot be ignored. The advantage of using differences between deviations, rather than the deviations themselves, is that this cancels out any curvature due to factors other than the orientation of the bars, which may also influence the path. For instance, in Fig. 5A we see a tendency for the hand to curve slightly away from the subject. We do not know the origin of this curvature, but by comparing half-way deviations we implicitly assume that it is independent of the curvature induced by the oriented bar. Whether this assumption is justified remains to be seen, but at present this assumption is necessary to be able to compare the movements with the perceptual judgements in any quantitative manner.

The half-way deviation is certainly not the only possible measure for cancelling out curvature due to factors other than the bar's orientation. We chose it because it is simple, but we could just as well have chosen a different measure, such as the area between the paths. This choice is not completely unimportant. In comparison with the half-way deviation, taking the area between the paths would increase the quantitative estimate of the influence of the bar's orientation for minimal jerk trajectories, relative to the estimate for constant curvature trajectories. Whether this is an improvement depends on how the curvature arises.

We concluded that subjects primarily misjudge the direction in which they have to move to reach the target. The curvature of the path later in the movement results from the adjustments that are needed to arrive at the target. The combination of starting systematically in the wrong direction and then correcting the path in order to arrive at the target gives rise to the tendency to approach the target orthogonally to the bar. If this scheme is correct then the bar's orientation primarily influences the beginning of the movement, so that a measure that is a direct consequence of 


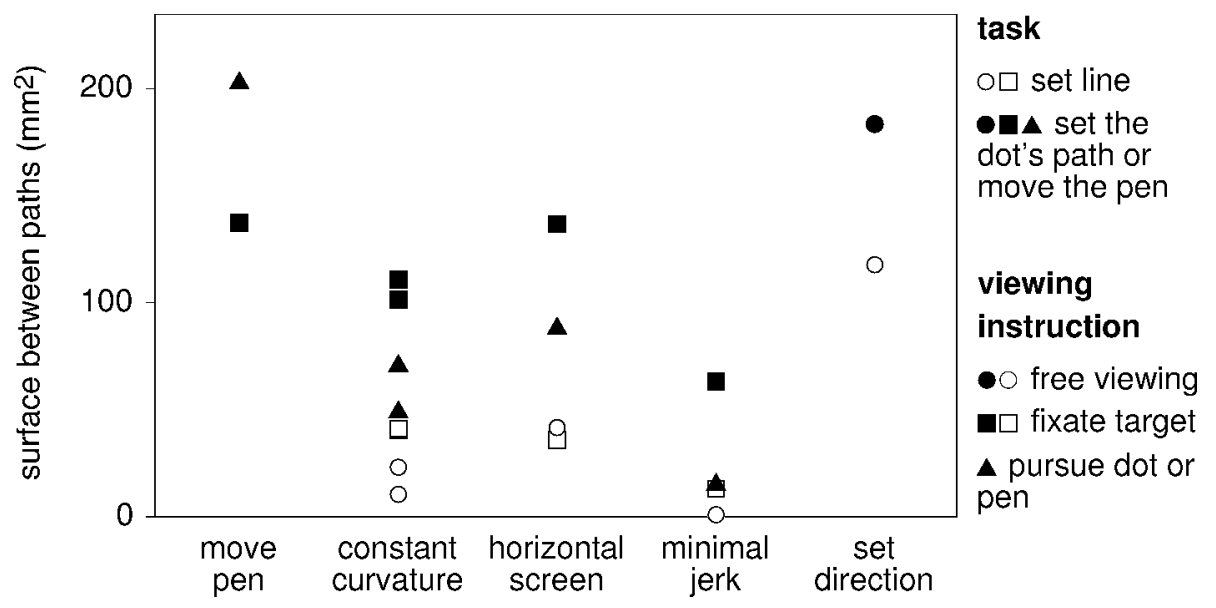

Figure 6. The surface between the paths toward the two oppositely slanted bars for each task and viewing instruction in Experiments $2 \mathrm{~A}$ to $2 \mathrm{E}$. Each symbol shows the average of 10 subjects.

the initial direction of movement, such as the deviation at a fixed distance from the start, is most appropriate. However, there is obviously some circular reasoning here, because we derived this scheme on the basis of such a measure: the half-way deviation. We therefore decided to examine whether our conclusions are likely to have been different if we had chosen a different measure. To do so we determined the average area between the paths.

Figure 6 shows the average area between the paths towards the targets on upward and downward oriented bars in Experiments 2A to 2E. This corresponds with the area between the thick lines in Fig. 5. The leftmost symbols show the surface between the average pen paths for the two oppositely slanted bars (move pen; Experiment 2A). The surface was slightly larger when subjects pursued the pen. The next set of symbols show the area between the constant curvature lines and dot paths for each viewing condition for the vertical screen (constant curvature; Experiments $2 \mathrm{~B}$ and 2D). The two values for each condition were very similar. Only the values for setting the dot's path while fixating the target came close to the values for the pen movements. Viewing the bar and targets at an angle on a horizontal screen (Experiment 2C), approximately as one saw them when moving the pen, increased the surface slightly. The surface for setting the dot's path while fixating the target was now almost as large as that for the pen movements that were made while fixating the target. However making the paths more realistic (minimal jerk; Experiment 2D) decreased the surface considerably, irrespective of the task and viewing condition, which suggests that this similarity was misleading. If only the first part of the more realistic paths is shown, the surface between the paths (including the invisible parts) is about as large as for the pen movements (set direction; Experiment 2E). Thus using the surface between the paths as our measure would lead to the same conclusion. 


\section{CONCLUSION}

We conclude that the influence of target orientation on the hand's path is mediated by a misperception of the direction to the target. In accordance with a perceptual origin, the velocity of the hand's movement hardly affected the target's influence. The target's position in space, and where the subject was looking during the movement, was also rather unimportant. The perceptual judgements were more sensitive to experimental variations, presumably because they could be improved by information which was not available for the movement. The notion that systematic misperceptions can make our hand move along a curved path from one position to another is not new (De Graaf et al., 1991; Wolpert et al., 1994). This has been interpreted to indicate that the hand's path is planned in visually (mis)perceived space (Flanagan and Rao, 1995; Miall and Haggard, 1995). We here show that this need not be a general misperception of space, but could be limited to a misperception of the direction in which the hand should move to reach the target.

\section{REFERENCES}

Abrams, R. A. and Landgraf, J. Z. (1990). Differential use of distance and location information for spatial localization, Percept Psychophys 47, 349-359.

Barreca, D. M. and Guenther, F. H. (2001). A modeling study of potential sources of curvature in human reaching movements, J. Motor Behavior 33, 387-400.

Boessenkool, J. J., Nijhof, E.-J. and Erkelens, C. J. (1998). A comparison of curvatures of left and right hand movements in a simple pointing task, Experimental Brain Research 120, 369-376.

Brenner, E., van den Berg, A. V. and van Damme, W. J. (1996). Perceived motion in depth, Vision Research 36, 699-706.

Brenner, E. and Cornelissen, F. W. (2000). Separate simultaneous processing of egocentric and relative positions, Vision Research 40, 2557-2563.

Brenner, E. and Smeets, J. B. J. (1995). Moving one's finger to a visually specified position: target orientation influences the finger's path, Experimental Brain Research 105, 318-320.

Fitts, P. M. and Peterson, J. R. (1964). Information capacity of discrete motor responses, J. Exper. Psychol. 67, 103-112.

Flanagan, J. R. and Rao, A. K. (1995). Trajectory adaptation to a nonlinear visuomotor transformation: evidence of motion planning in visually perceived space, J. Neurophysiol. 74, 2174-2178.

Flash, T. and Hogan, N. (1985). The coordination of arm movements: an experimentally confirmed mathematical model, J. Neurosci. 5, 1688-1703.

Gillam, B. and Chambers, D. (1985). Size and position are incongruous: measurements on the Müller-Lyer figure, Perception and Psychophysics 37, 549-556.

Goodbody, S. J. and Wolpert D. M. (1999). The effect of visuomotor displacements on arm movement paths, Experimental Brain Research 127, 213-223.

De Graaf, J. B., Sittig, A. C. and Denier van der Gon, J. J. (1991). Misdirections in slow goal-directed arm movements and pointer-setting tasks, Experimental Brain Research 84, 434-438.

De Graaf, J. B., Sittig, A. C. and Denier van der Gon, J. J. (1994). Misdirections in slow, goal-directed arm movements are not primarily visually based, Experimental Brain Research 99, 464-472.

De Graaf, J. B., Denier van der Gon, J. J. and Sittig, A. C. (1996). Vector coding in slow goal-directed arm movements, Perception and Psychophysics 58, 587-601.

Mack, A., Heuer, F., Villardi, K. and Chambers, D. (1985). The dissociation of position and extent in Müller-Lyer figures, Perception and Psychophysics 37, 335-344. 
MacKay, D. and Newbigging, P. L. (1977). The Poggendorff and its variants do arouse the same perceptual process, Perception and Psychophysics 21, 26-32.

Miall, R. C. and Haggard, P. N. (1995). The curvature of human arm movements in the absence of visual experience, Experimental Brain Research 103, 421-428.

Mohrmann-Lendla, H. and Fleischer, A. G. (1991). The effect of a moving background on aimed hand movements, Ergonomics 34, 353-364.

Nakano, E., Imamizu, H., Osu, R., Uno, Y., Gomi, H., Yoshioka, T. and Kawato, M. (1999). Quantitative examinations of internal representations for arm trajectory planning: minimum commanded torque change model, J. Neurophysiol. 81, 2140-2155.

Nishikawa, K. C., Murray, S. T. and Flanders, M. (1999). Do arm postures vary with the speed of reaching? J. Neurophysiol. 81, 2582-2586.

Osu, R., Uno, Y., Koike, Y. and Kawato, M. (1997). Possible explanations for trajectory curvature in multi-joint arm movements, J. Exper. Psychol.: Human Perception and Performance 23, 890-913.

Smeets, J. B. J. and Brenner, E. (1999). A new view on grasping, Motor Control 3, 237-271.

Van Thiel, E., Meulenbroek R. G. J. and Hulstijn, W. (1998). Path curvature in workspace and in joint space: Evidence for coexisting coordinative rules in aiming, Motor Control 2, 331-351.

Wolpert, D. M., Ghahramani, Z. and Jordan, M. I. (1994). Perceptual distortion contributes to the curvature of human reaching movements, Experimental Brain Research 98, 153-156. 